\title{
Intra-Cavity Spectroscopy Using Amplified Spontaneous Emission in Fiber Lasers
}

\author{
Norhana Arsad, Min Li, George Stewart, and Walter Johnstone
}

\begin{abstract}
Fiber laser sources offer interesting possibilities for gas sensors since they can operate over an extended wavelength range, encompassing the near-IR absorption lines of a number of important gases but a major problem is that overtone absorption lines of gases in the near-IR are relatively weak. In order to enhance sensitivity, we present here a simple method of intra-cavity absorption spectroscopy (ICAS) which makes use of the amplified spontaneous emission (ASE) already present within a fiber laser cavity. The ASE also provides a convenient broadband source for the simultaneous interrogation of several gases within the gainbandwidth of the fiber laser. The key principle is based on adjusting the cavity attenuation to select an appropriate inversion level where the fiber gain curve is flat. Under this condition, the ASE undergoes multiple circulations within the fiber laser cavity, enhancing the effective path-length of a gas cell placed within the laser cavity. A theoretical model of system operation is given and we have experimentally demonstrated the principle of operation with acetylene and carbon dioxide using a simple erbium fiber laser system containing a $6 \mathrm{~cm}$ path-length, fiber coupled, intra-cavity, micro-optic gas cell. We have experimentally simultaneously observed 16 absorption lines for $1 \%$ acetylene gas in the $1530 \mathrm{~nm}$ region and detected the very weak carbon dioxide lines in this same wavelength region. A path length enhancement of $\sim 500$ in the linear regime has been demonstrated transforming the $6 \mathrm{~cm}$ microoptic cell into an effective path length of $\sim 30 \mathrm{~m}$. We also demonstrate how the enhancement factor may be calibrated by use of a simple fiber-optic interferometer. Apart from the OSA, all components are inexpensive and the system is very simple to construct and operate.
\end{abstract}

Index Terms-Intra-cavity spectroscopy, laser spectroscopy, erbium-doped fiber lasers, fiber sensors, gas sensors.

\section{INTRODUCTION}

G AS sensing is very important for environmental, safety and industrial monitoring. Optical fiber gas sensors operating in the near-IR region (1-2 $\mu \mathrm{m}$ wavelength) have several advantages, including the availability of low cost components,

Manuscript received September 27, 2010; revised December 08, 2010; accepted December 15, 2010. Date of publication December 30, 2010; date of current version March 02, 2011. This work was supported by the Ministry of Higher Education, Malaysia, and Universiti Kebangsaan Malaysia.

N. Arsad is with the Centre for Microsystems and Photonics, Department of Electronic and Electrical Engineering, University of Strathclyde, Glasgow, G1 1XW, U.K., and also with the Department of Electrical, Electronic and Systems, Universiti Kebangsaan Malaysia, Bangi Selangor, Malaysia.

M. Li is with the Centre for Microsystems and Photonics, Department of Electronic and Electrical Engineering, University of Strathclyde, Glasgow, G1 1XW, U.K., and also with the Department of Physics, Wuhan University of Technology, 430072 Wuhan, China.

G. Stewart and W. Johnstone are with the Centre for Microsystems and Photonics, Department of Electronic and Electrical Engineering, University of Strathclyde, Glasgow, G1 1XW, U.K.

Color versions of one or more of the figures in this paper are available online at http://ieeexplore.ieee.org.

Digital Object Identifier 10.1109/JLT.2010.2103048 remote access to hazardous environments and networking capabilities. Fiber laser sources offer interesting possibilities for gas sensors [1] since they can operate over an extended wavelength range and micro-optic gas cells may be readily connected either external to, or within, the fiber laser cavity. Erbium fiber lasers can operate over the wavelength range of $\sim 1480-1620$ $\mathrm{nm}$, encompassing the near-IR absorption lines of gases such as $\mathrm{CO}, \mathrm{CO}_{2}, \mathrm{C}_{2} \mathrm{H}_{2}, \mathrm{H}_{2} \mathrm{~S}, \mathrm{NH}_{3}, \mathrm{OH}, \mathrm{H}_{2} \mathrm{O}, \mathrm{HI}$ and other fiber dopants or Raman fiber lasers extend the available wavelength range even further to include $\mathrm{C}_{2} \mathrm{H}_{4}$ and $\mathrm{C}_{2} \mathrm{H}_{6}$, etc. However a major problem is that the overtone absorption lines of gases in the near-IR are typically two or three orders of magnitude weaker than their fundamental lines in the midinfrared. One method to enhance sensitivity is use of the technique known as "intra-cavity laser absorption spectroscopy" (ICLAS) where the gas cell is placed within a laser cavity and the evolution of the output spectrum is captured over the build-up period of laser oscillation in the cavity. Enhancement of the effective path length of the gas cell occurs due to the multiple circulations of light within the cavity during this period. The technique has been successfully demonstrated with solid-state lasers [2] and effective absorption path lengths ranging from tens to thousands of kilometers, depending on the laser type and operation conditions, have been attained. We have previously reported a theoretical analysis of the dynamics of erbium fiber laser systems [3], [4] but practical realization of ICLAS with fiber lasers is difficult for several reasons - the spectrum must be captured during the period of relaxation oscillations and detector arrays for the $1-\mu \mathrm{m}$ region are less capable and more expensive than the silicon CCD arrays used for ICLAS at $<1 \mu \mathrm{m}$ wavelengths. For example, Stark [5] used a rapid scan Fourier Transform (FT) spectrometer to capture the spectrum at each peak of the relaxation oscillations for a thulium-doped fiber laser.

In this paper we present a simple and inexpensive method of intra-cavity absorption spectroscopy (ICAS) which makes use of the amplified spontaneous emission (ASE) already present within the fiber laser cavity and we experimentally demonstrate the principle of operation using acetylene gas, with a path length enhancement of $\sim 500$.

\section{THEORETICAL DESCRIPTION OF ICAS USING ASE IN LASER CAVITY}

\section{A. Key Principles of ICAS With ASE in a Fiber Laser Cavity}

The first key principle for attaining path length enhancement within a fiber laser cavity is to ensure a broad, flat erbium gain spectrum, which preferable can be tuned over the desired spectral regions where particular gas absorption lines exist. The gain characteristics of fiber amplifiers and lasers may 


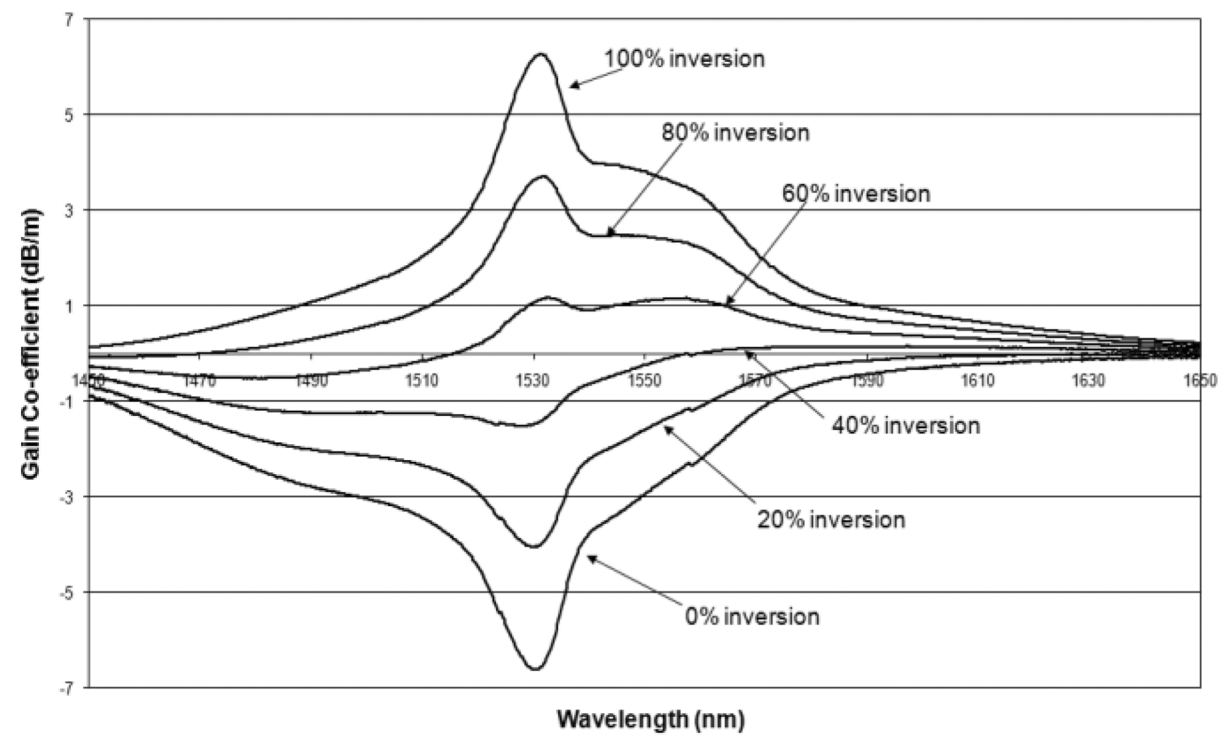

Fig. 1. Length-averaged gain coefficient of erbium-doped fiber for different inversion levels.

be conveniently described in terms of a length averaged gain coefficient, $\bar{g}$, given by [1], [4], [6]:

$$
\bar{g}=(\gamma+\alpha) \bar{N}_{2}-\alpha
$$

where $\gamma$ is the emission coefficient, $\alpha$ is the absorption coefficient and $\bar{N}_{2}$ is the length-averaged inversion level $\left(0<\bar{N}_{2}<\right.$ 1) where $\bar{N}_{2}=(1 / l) \int_{0}^{l} N_{2}(z) d z$. The total gain, $G$, of a length, $l$, of erbium fiber is given by: $G=\bar{g} l$.

Fig. 1 shows $\bar{g}$ as a function of wavelength for different inversion levels, $\bar{N}_{2}$, in erbium-doped fiber as used in our erbium fiber laser systems. It is clear from Fig. 1 that the shape of the gain spectrum is dependent on the inversion level and a reasonably flat spectrum is attained around the $1530 \mathrm{~nm}$ and $1560 \mathrm{~nm}$ regions when the inversion level is $\sim 60 \%$ and $\bar{g} \approx 1$ $\mathrm{dB} / \mathrm{m}$. When the cavity is lasing, the inversion level is determined by the external cavity loss since steady-state laser oscillation implies that gain and loss are (almost) equally balanced (net round-trip gain of approximately unity). For example, with a cavity loss of $10 \mathrm{~dB}$, then an erbium fiber gain of $G=+10 \mathrm{~dB}$ is required for lasing, which means that $\bar{g} \approx 1 \mathrm{~dB} / \mathrm{m}$ for a typical erbium fiber length of $10 \mathrm{~m}$ and hence the system operates at $60 \%$ inversion level. Increasing or decreasing the cavity loss will correspondingly increase or decrease the inversion level, so a variable attenuator within the fiber laser cavity may be used to select a particular gain curve in Fig. 1. Similarly, increasing the erbium length will reduce the inversion level. Assuming homogeneous broadening is dominant, the actual lasing wavelength is determined by the position of the local maximum on the appropriate gain curve in Fig. 1 and the lasing wavelength may be switched between $\sim 1530 \mathrm{~nm}$ and $1560 \mathrm{~nm}$ by simple adjustment of the variable attenuator to raise or lower the inversion level around $60 \%$.

The second key principle relates to the characteristics of the ASE within the fiber laser cavity. ASE exists over the gain-bandwidth of the doped fiber and provides a convenient broadband source for interrogation of gas absorption lines. As noted above, the net round-trip gain is approximately unity at the lasing wavelength. For the ASE near the lasing wavelength, the round-trip gain is slightly less than, but close to, unity if the gain curve is flat. Hence, the ASE undergoes multiple circulations within the fiber laser cavity and consequently the effective path-length of an intra-cavity gas cell is greatly increased.

\section{B. Theoretical Description of ASE and ICAS in a Fiber Laser Cavity}

The total ASE emerging from one end of an erbium-doped fiber of length $l$ over a frequency bandwidth $\Delta \nu=-\left(c / \lambda^{2}\right) \Delta \lambda$ is given by [4]

$$
P_{\mathrm{ASE}}(\lambda)=2 \gamma l \cdot \bar{N}_{2} \cdot A \cdot \Delta \nu \cdot\left(\frac{h c}{\lambda}\right)
$$

where $A=\left(e^{\bar{g} l}-1\right) / \bar{g} l$ represents the amplification of the spontaneous emission (SE) over the fiber length.

When a fiber laser cavity is formed (see Fig. 2), the ASE makes multiple circulations in the cavity and the total ASE, $P_{t}(\lambda)$, emerging at the erbium-doped fiber output is described by the geometric series

$$
\begin{aligned}
P_{t}(\lambda)=P_{\mathrm{ASE}}(\lambda)+\left\{e^{-\alpha_{c}} \cdot e^{\bar{g} l} \cdot e^{-\alpha_{g} C L}\right\} P_{\mathrm{ASE}}(\lambda) \\
+\left\{e^{-\alpha_{c}} \cdot e^{\bar{g} l} \cdot e^{-\alpha_{g} C L}\right\}^{2} P_{\mathrm{ASE}}(\lambda)+\cdots
\end{aligned}
$$

where $\alpha_{c}$ is the cavity loss factor, $\alpha_{g}$ the gas absorption coefficient, $C$ the gas concentration and $L$ the absorption path length of the gas cell within the cavity.

Summing the series of (3) to infinity, the ASE power coupled out of the loop by a fiber coupler with output coupling ratio $R_{o}$ is

$$
P_{o}(\lambda)=\frac{R_{o} \cdot P_{\mathrm{ASE}}(\lambda)}{1-e^{-\delta} \cdot e^{-\alpha_{g} C L}}
$$

where $\delta(\lambda)=\alpha_{c}-\bar{g} l$ is the difference between the cavity loss and the total gain of the erbium fiber length. 


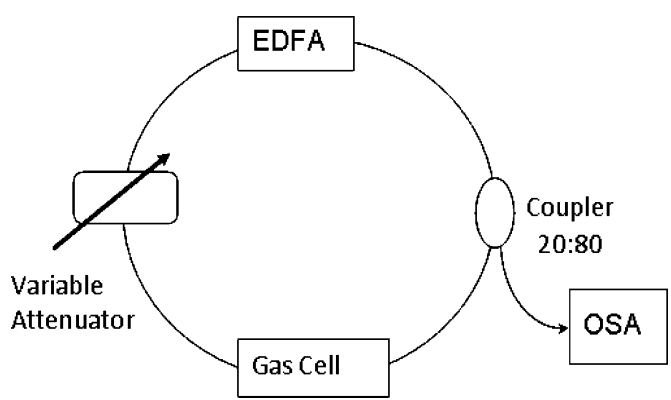

Fig. 2. Experimental system for ICAS using ASE.

If the cavity is near or above threshold for lasing and the gain curve is flat, then $\delta$ will be small in the vicinity of the lasing wavelength so we may write $e^{-\delta} \approx 1-\delta$ and if the gas absorbance is also small then $e^{-\alpha_{g} C L} \approx 1-\alpha_{g} C L$. The ratio of the output ASE with gas, $P_{g}$, to the background, $P_{\mathrm{bg}}$, without gas in the cell, is then given by

$$
\frac{P_{g}(\lambda)}{P_{\mathrm{bg}}(\lambda)} \cong \frac{1}{\left(1+\alpha_{g} C L / \delta(\lambda)\right)}
$$

Equation (5) indicates that the gas absorption observed in the ASE signal is non-linearly dependent on the concentration (in a different manner from the non-linear exponential form of the Beer-Lambert absorption law) and is enhanced through the factor $\delta(\lambda)$. The enhancement increases near the lasing wavelength as $\delta(\lambda)$ pproaches zero (although $\delta$ does not actually reach zero [4] even at the lasing wavelength). The effective enhancement is greatest for very weak absorption or very low concentrations where $\alpha_{g} C L / \delta(\lambda) \ll 1$ and the linear approximation to (5) is valid:

$$
\frac{P_{g}(\lambda)}{P_{\mathrm{bg}}(\lambda)} \cong\left(1-\alpha_{g} C L / \delta(\lambda)\right)
$$

This may be compared to the linear approximation to the Beer-Lambert absorption law, $P_{\text {out }} / P_{\text {in }}=e^{-\alpha_{g} C L_{\text {eff }}} \approx$ $\left(1-\alpha_{g} C L_{\text {eff }}\right)$ for a single pass cell, from which we may define an effective path length for the linear regime of the ICAS as $L_{\text {eff }}=L / \delta(\lambda)$. For $\delta<0.01 \mathrm{~dB}$, the path length enhancement, $L_{\text {eff }} / L$, in the linear regime is $>400$.

\section{EXPERIMENTAL DEMONSTRATION OF ICAS USING ASE IN A FIBER LASER}

The experimental system for demonstration of ICAS using ASE is shown in Fig. 2. The erbium doped fiber amplifier (EDFA) unit (OptoSci Ltd., $\sim 1.8 \mathrm{~dB}$ internal loss) consists of $\sim 10.9 \mathrm{~m}$ of erbium-doped fiber, pumped by a $980 \mathrm{~nm}$ diode laser with two wavelength division multiplexers for in/out coupling of the pump and an isolator at both the input and the output of the EDFA. A $6 \mathrm{~cm}$ path-length micro-optic gas cell (insertion loss of $\sim 1 \mathrm{~dB}$ ) [7] is fiber-coupled within the cavity and a variable attenuator (EXFO, Optical Test System IQ-203, insertion loss of $\sim 3 \mathrm{~dB}$ ) is also included within the cavity to adjust the inversion levels as discussed above. A 20:80 single mode fiber coupler is used to tap off $20 \%$ of the cavity light to an optical spectrum analyzer (OSA: Agilent 86140B resolution

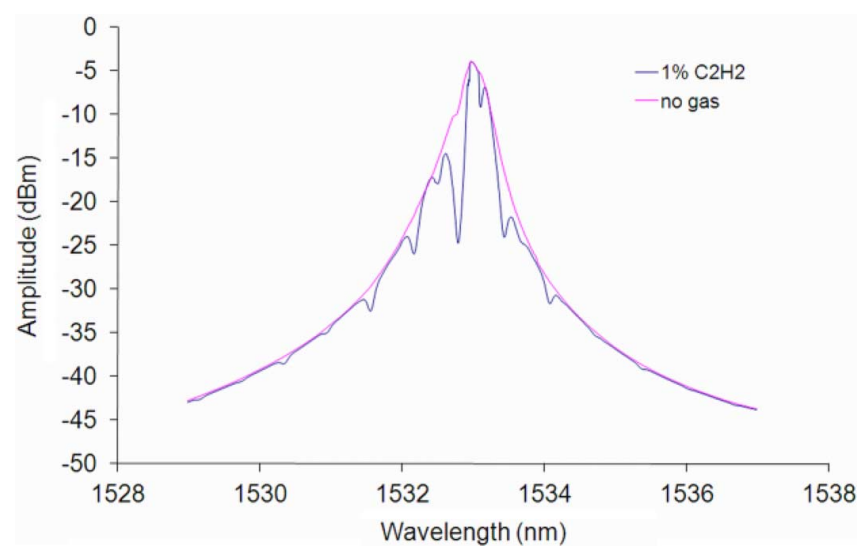

Fig. 3. ASE output with and without $1 \% \mathrm{C}_{2} \mathrm{H}_{2}$ in the intra-cavity gas cell.

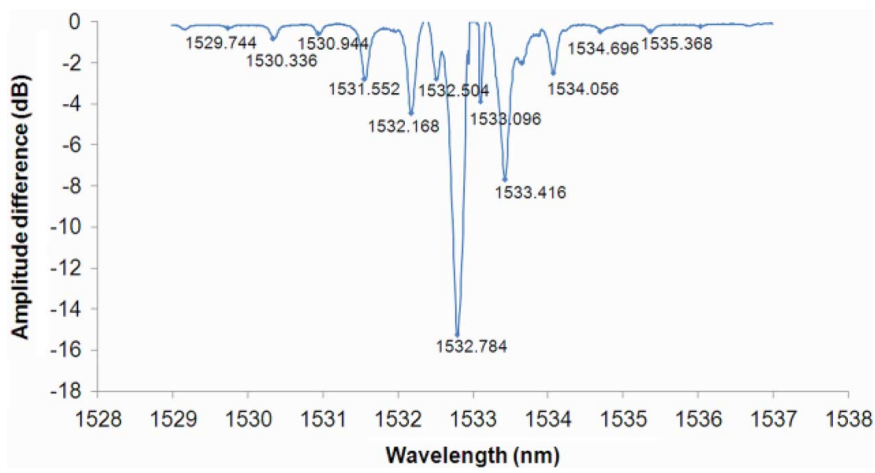

Fig. 4. $\mathrm{C}_{2} \mathrm{H}_{2}$ lines after background subtraction.

of $0.06 \mathrm{~nm}$ ) to monitor the cavity ASE and the laser emission. Total cavity length is $\sim 13 \mathrm{~m}$ and all connectors are FC/APC except for the variable attenuator which had $\mathrm{FC} / \mathrm{PC}$ connectors.

Compared with other hydrocarbon gases, acetylene $\left(\mathrm{C}_{2} \mathrm{H}_{2}\right)$ has relatively strong lines in the near-IR around $1530 \mathrm{~nm}$ and hence measurements were performed using $1 \% \mathrm{C}_{2} \mathrm{H}_{2}$ in the micro-optic gas cell to evaluate the performance of the system. Fig. 3 shows the ASE output observed on the OSA over the wavelength range of $1529-1537 \mathrm{~nm}$ both with $1 \% \mathrm{C}_{2} \mathrm{H}_{2}$ in the gas cell and after the cell was flushed with nitrogen (no gas background) for a pump power of $70.2 \mathrm{~mW}$ and an added attenuation of $11.8 \mathrm{~dB}$. With this pump power the cavity is above threshold and the inversion level clamped by the laser action. (For clarity in Fig. 3, the lasing peak at $1533 \mathrm{~nm}$ has been removed to show the ASE distribution).

A total of 16 acetylene absorption lines can be observed in Fig. 3 and, in particular, the lines near the central (lasing region) are greatly enhanced in amplitude. This is illustrated more clearly in Fig. 4 where the background ASE curve, $P_{\mathrm{bg}}$, has been subtracted from the gas line $\left(P_{g}-P_{\mathrm{bg}}\right.$ in units of $\left.\mathrm{dB}\right)$ and the central absorption line at $1532.792 \mathrm{~nm}$ appears with $\sim 15 \mathrm{~dB}$ of attenuation. In order to compare the results with theory and obtain the enhancement factor, the $\mathrm{dB}$ scale of Fig. 4 is converted to relative transmission, $P_{g} / P_{\mathrm{bg}}$, in Fig. 5 using the relationship

$$
P_{g} / P_{\mathrm{bg}}=10^{\left(\frac{P_{g}(\mathrm{dBm})_{-P_{\mathrm{bg}}}(\mathrm{dBm})_{)}}{10}\right) .}
$$




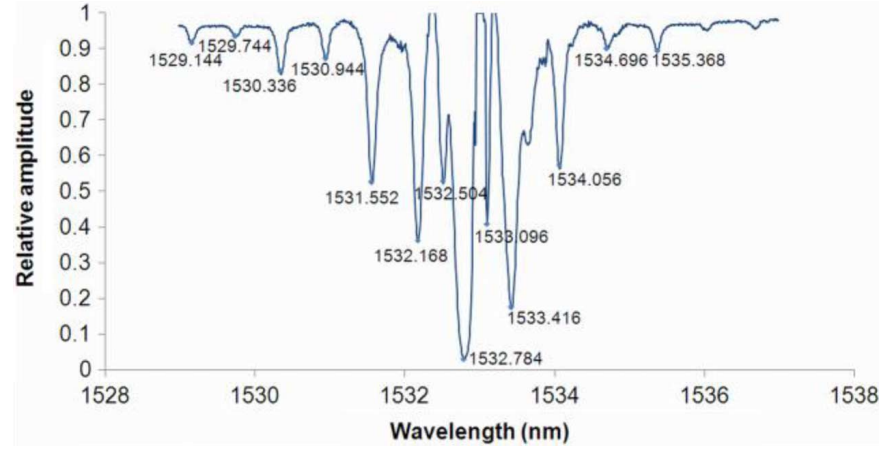

Fig. 5. Relative transmission of $1 \% \mathrm{C}_{2} \mathrm{H}_{2}$ obtained experimentally.

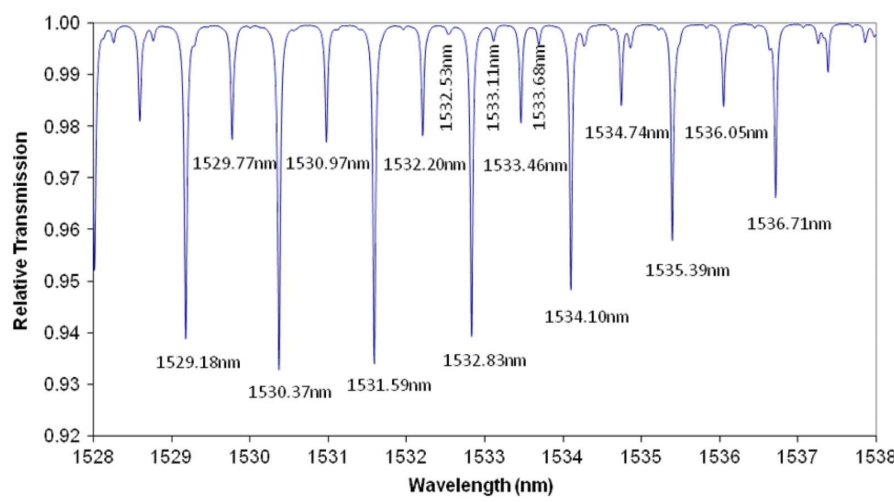

Fig. 6. Theoretical absorption lines computed for $1 \% \mathrm{C}_{2} \mathrm{H}_{2}$ and a $6 \mathrm{~cm}$ path length. (HITRAN 2008 database).

Fig. 6 shows the theoretical acetylene absorption lines, calculated from the HITRAN database 2008, using commercial software (GATS Spectral Calculator) to calculate the relative transmission for $1 \% \mathrm{C}_{2} \mathrm{H}_{2}$ with a $6 \mathrm{~cm}$ path-length (micro-optic cell path length). All 16 acetylene lines observed experimentally are identified on the theoretical spectrum allowing for a small calibration error on the OSA wavelength scale.

Figs. 5 and 6 may be compared to obtain the enhancement factor. For example, for the central absorption line at 1532.792 $\mathrm{nm}$ in Fig. 5, the theoretical relative transmission for the corresponding absorption line in Fig. 6 at $1532.8296 \mathrm{~nm}$ is $94 \%$ for $1 \% \mathrm{C}_{2} \mathrm{H}_{2}$ over a $6 \mathrm{~cm}$ path length. Using these figures in the Beer-Lambert Law, $P_{\text {out }} / P_{\text {in }}=\exp \left[-\alpha_{g}(\nu) C L\right]$, we obtain the line center absorbance, $A=\alpha_{g} C L=0.06$ for these conditions. Fig. 5 shows that under the same conditions, the experimental relative transmission observed with the ICAS system for this line is $P_{g} / P_{\mathrm{bg}}=0.03$ (or 3\%). Using these values in (5) gives $\delta \sim 0.002(0.00868 \mathrm{~dB})$ or an effective path length enhancement of 500 in the linear regime.

As noted in the theoretical section from (5) and (6), ICAS operates most effectively in, or near, the linear regime where the gas concentration and/or the absorption coefficient is very weak. This effect can be clearly seen from Figs. 5 and 6. In Fig. 6, the lines on either side of the $1532.83 \mathrm{~nm}$ line at 1532.53 and $1533.11 \mathrm{~nm}$ are much weaker than the central $1532.83 \mathrm{~nm}$ line but clearly appear with relatively strong absorption in Fig. 5 . The 1532.53 and $1533.11 \mathrm{~nm}$ lines have a relative transmission

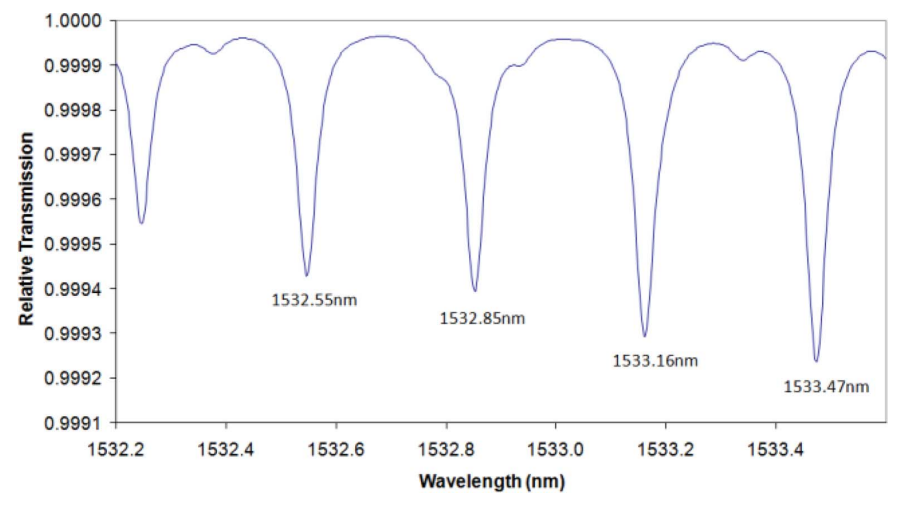

Fig. 7. Theoretical absorption lines computed for $100 \% \mathrm{CO}_{2}$ and $6 \mathrm{~cm}$ path length cell. (HITRAN 2008 database).

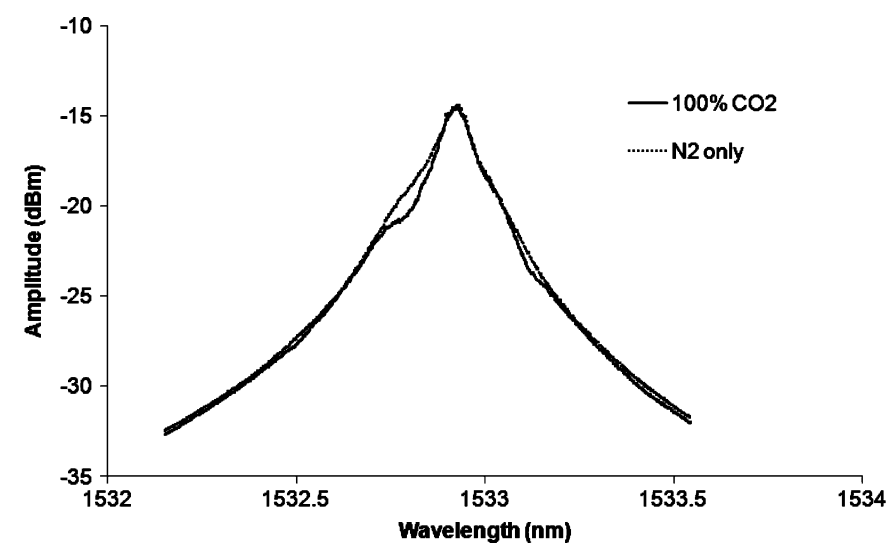

Fig. 8. Measured ASE spectrum with and without $100 \% \mathrm{CO}_{2}$ in the intracavity gas cell.

of $99.78 \%$ and $99.64 \%$, respectively, from the HITRAN database but appear in Fig. 5 with a relative transmission of $50 \%$ and $40 \%$ respectively. Using these values in (5) gives similar values of $\delta \sim 0.0022$ and $\delta \sim 0.0024$ respectively, corresponding to effective path length enhancements of $\sim 450$ and 400 in the linear regime.

To directly demonstrate the ability of the ICAS system to detect weak absorption lines, we have detected the very weak $\mathrm{CO}_{2}$ lines in the $1530 \mathrm{~nm}$ region. Fig. 7 shows the theoretical absorption lines over the $1532-1534 \mathrm{~nm}$ region from the $\mathrm{HI}-$ TRAN 2008 database for $100 \% \mathrm{CO}_{2}$ and $6 \mathrm{~cm}$ path length. The $\mathrm{CO}_{2}$ absorption line at $1532.85 \mathrm{~nm}$ has a relative transmission of $99.94 \%$ on the peak. Fig. 8 shows the measured ASE spectrum from the ICAS system with and without $100 \% \mathrm{CO}_{2}$ in the cell and Fig. 9 shows the difference $(\mathrm{dB})$ between the two curves of Fig. 8. Four $\mathrm{CO}_{2}$ absorption lines are identified (allowing for a calibration error of $0.05 \mathrm{~nm}$ on the OSA) and the $1532.8 \mathrm{~nm}$ $\mathrm{CO}_{2}$ line gives an attenuation of $\sim 1.2 \mathrm{~dB}$ on the peak. Using these values in (5) gives an enhancement factor of 530.

For the sensitivity and resolution in gas concentration measurements, the fundamental limit for the ICAS system is determined by the resolution in optical power measurement of the output spectrum. For the optical spectrum analyzer (Agilent $86140 \mathrm{~B}$ ) used in our system, the resolution is $\sim 0.05 \mathrm{dBm}$. Other noise factors such as vibration may limit the performance, but 


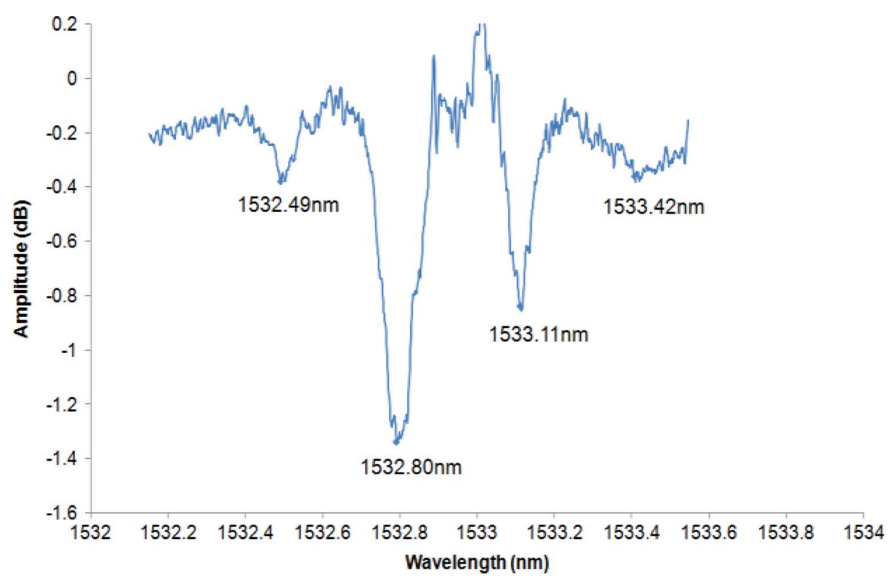

Fig. 9. $\mathrm{CO}_{2}$ absorption lines after background subtraction.

the low-noise traces as obtained in Figs. 3 and 8 indicate that a conservative value of $\sim 0.1 \mathrm{dBm}$ is readily attainable. Using (6) with this value of $0.1 \mathrm{dBm}$, the smallest absorbance that can be measured for an enhancement factor of $\sim 450$ is therefore

$$
\alpha_{g} C_{\min } L \cong(0.1) \delta / 4.34 \cong 5 \times 10^{-5} .
$$

With a cell length $L=6 \mathrm{~cm}$ and with $\alpha_{g} \sim 1 \mathrm{~cm}^{-1}$ for the $1532.83 \mathrm{~nm}$ line of $\mathrm{C}_{2} \mathrm{H}_{2}$ and $\alpha_{g} \sim 1 \times 10^{-4} \mathrm{~cm}^{-1}$ for the $1532.85 \mathrm{~nm}$ line of $\mathrm{CO}_{2}$ from Figs. 6 and 7 respectively, this translates to a sensitivity, $C_{\min } \sim 8 \mathrm{ppm}$ for $\mathrm{C}_{2} \mathrm{H}_{2}$ and $\sim 8 \%$ for $\mathrm{CO}_{2}$.

The resolution (smallest change in concentration, $\Delta C$, that can be detected) is also related to the power resolution measurement $(0.1 \mathrm{dBm})$ and may be determined from (5) as

$$
\alpha_{g}(\Delta C) L \cong \frac{(0.1) \delta}{4.34}\left(1+\frac{\alpha_{g} C L}{\delta}\right) .
$$

As (8) indicates, the resolution is dependent on the concentration and becomes poorer at high concentrations. Under conditions where the linear approximation, (6), is valid, $\alpha_{g} C L / \delta \ll$ 1 , then the resolution is equal to the sensitivity as discussed above.

\section{MODELING OF ICAS AND COMPARISON WITH EXPERIMENTAL RESULTS}

In order to model the experimental system the following parameters and experimental conditions were used. Data for the absorption and emission coefficients $(\alpha, \gamma)$ of the erbium-doped fiber as a function of wavelength (Fig. 1) were supplied by the manufacturer (Fibrecore Ltd) and, as noted, the erbium-doped fiber length was $10.9 \mathrm{~m}$. The cavity loss was adjusted by the variable attenuator in Fig. 2 to set the inversion level at 60\% which could be observed by simultaneous lasing around the $1530 \mathrm{~nm}$ and $1560 \mathrm{~nm}$ regions (equal gain as indicated in Fig. 1). Since the enhancement is determined through: $\delta(\lambda)=\alpha_{c}-\bar{g} l$, the cavity loss, $\alpha_{c}$, must be determined as precisely as possible which is difficult.

The cavity loss was estimated from the ratio of the experimental ASE power at two wavelengths using (7) below. The ASE power was measured at a pump power of $10.9 \mathrm{~mW}$ (just

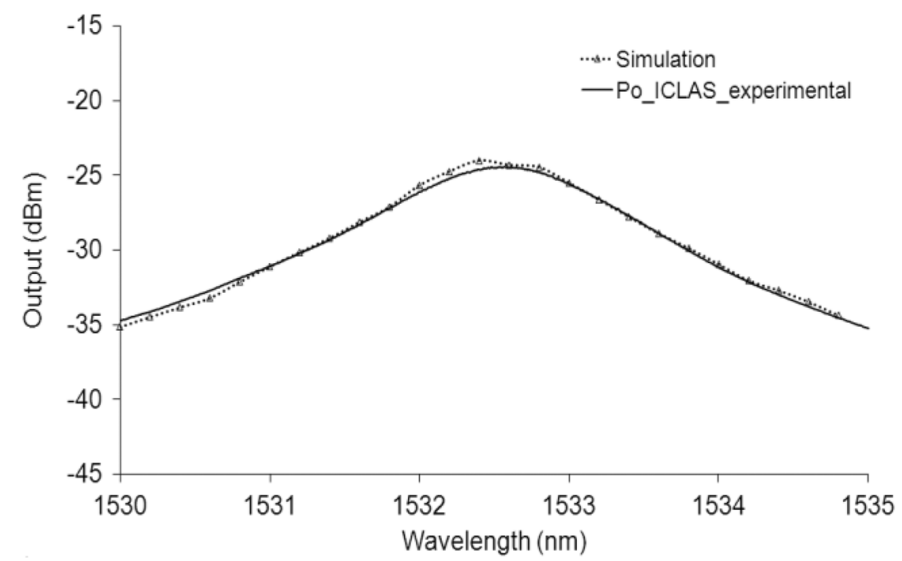

Fig. 10. Comparison between the experimental and theoretical ASE background.

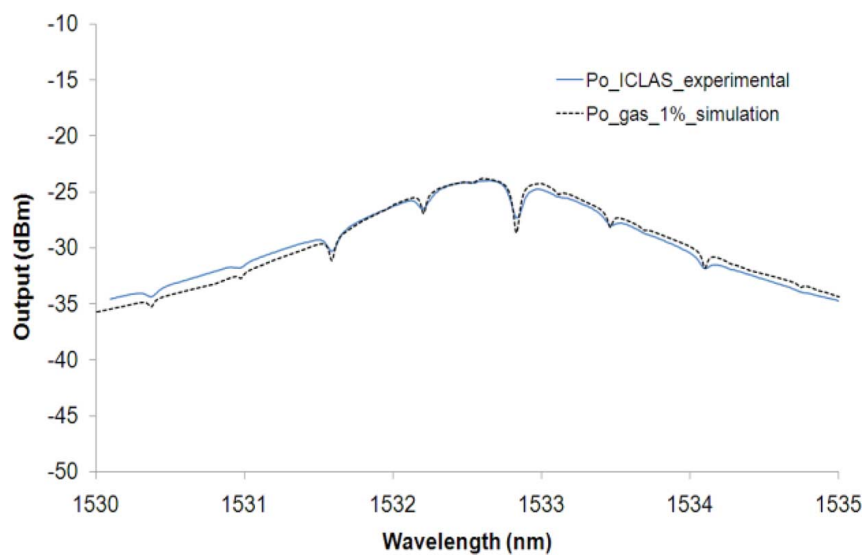

Fig. 11. Comparison between the experimental and theoretical ASE with $1 \%$ $\mathrm{C}_{2} \mathrm{H}_{2}$ in the gas cell.

below lasing threshold) at wavelengths of $1532.4 \mathrm{~nm}$ and 1532.8 $\mathrm{nm}$. From (2) and (4) this ratio is given by (without gas)

$$
\frac{P_{o}\left(\lambda_{1}\right)}{P_{o}\left(\lambda_{2}\right)}=\frac{\gamma_{1}}{\gamma_{2}} \cdot \frac{e^{g\left(\lambda_{1}\right) l}-1}{e^{g\left(\lambda_{2}\right) l}-1} \cdot \frac{g\left(\lambda_{2}\right)}{g\left(\lambda_{1}\right)} \cdot \frac{\alpha_{c}-g\left(\lambda_{2}\right) l}{\alpha_{c}-g\left(\lambda_{1}\right) l}
$$

Equation (9) can then be used, with the other known parameters, to calculate the cavity loss $\left(\alpha_{c}\right)$ as $13.49 \mathrm{~dB}$. With all the above parameters, an output coupling ratio $R_{o}$ of $20 \%, \Delta \nu=10 \mathrm{MHz}$ and an inversion level of $60.5 \%$, the ASE spectrum with and without gas may be simulated from (4).

Fig. 10 shows the ASE background at a pump power of $10.9 \mathrm{~mW}$ (just below the lasing threshold) and the close agreement between the theoretical curve calculated using (2) and (4) and the experimental distribution, allowing for an excess loss of $2.7 \mathrm{~dB}$ between the fiber laser output and the OSA from connectors, etc.

In Fig. 11 we simulate the expected ASE spectrum when gas is present by including the $\mathrm{C}_{2} \mathrm{H}_{2}$ gas absorption lines from the Hitran database (for $1 \%$ concentration and $6 \mathrm{~cm}$ gas cell length) in (4). Also shown in Fig. 11 are the experimental results under the same conditions. In Fig. 12 we have subtracted the theoretical and experimental background ASE (Fig. 10) from Fig. 11 to show the enhanced theoretical and experimental absorption lines. Also shown in Fig. 12 for comparison are the same absorption lines from the Hitran database. The general trend of 


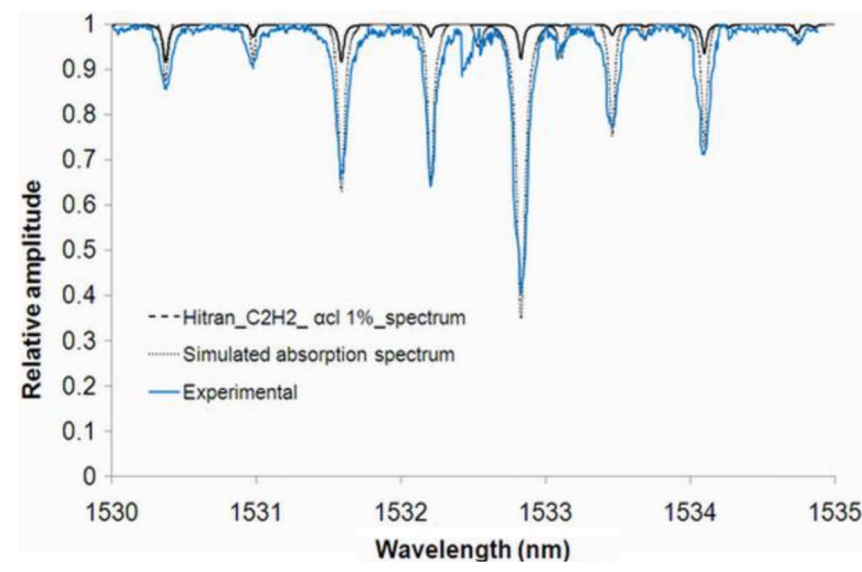

Fig. 12. Comparison between the experimental and theoretical ASE for $1 \%$ $\mathrm{C}_{2} \mathrm{H}_{2}$ with the background subtracted.

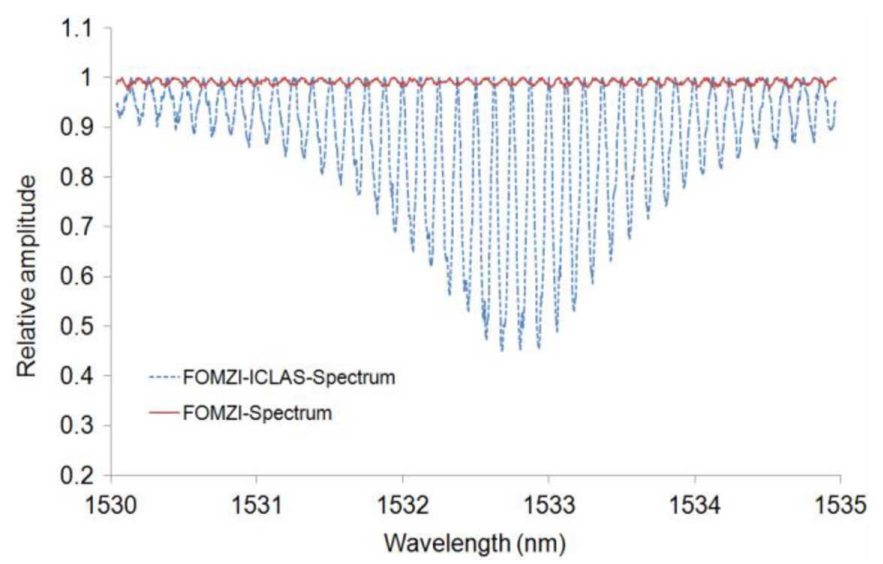

Fig. 13. Transmission of fiber Mach-Zehnder interferometer (solid line) and fringe enhancement when placed within the fiber cavity.

the theoretical simulation fits well with the experimental results although there are small differences between the experimental and theoretical enhancement observed on the some of the absorption line peaks, possibly due to the error in predicting the precise value of the cavity loss.

\section{CAlibration of the Enhancement Factor}

Since the enhancement factor is very sensitive to $\delta$ and hence is dependent on how close the absorption line is to the lasing wavelength, it is important for practical applications that some form of calibration be included in the system operation. Here we demonstrate the use of a fiber optic Mach-Zehnder interferometer (FOMZI) within the fiber loop cavity for this purpose.

The experimental FOMZI consists of two fiber couplers (a 90:10 coupler and a 99:1 coupler) to generate a small uniform fringe pattern across the wavelength region of interest. With a length difference of $\sim 1.25 \mathrm{~cm}$ between the two fiber arms, the fringe spacing is $\sim 0.13 \mathrm{~nm}(16.5 \mathrm{GHz})$. The transmission of the FOMZI was measured before being placed within the fiber loop cavity from the ratio of output to input power using the ASE from the EDFA as a convenient broadband source. The solid line in Fig. 13 shows the experimentally measured normalized transmission of the FOMZI with the $\sim 0.04 \mathrm{~dB}$ fringe pattern ripple. When the FOMZI is now placed within the ICAS loop, the fringe ripple spectrum is enhanced as shown by the dashed line in Fig. 13 and clearly illustrates quantitatively the variation in enhancement ( 10 to 130 here for $10.9 \mathrm{~mW}$ pump power) across the wavelength region of interest for a particular set of experimental conditions. Hence, the FOMZI, or similar calibration tool, may be included along with the gas absorption cell within the fiber loop to experimentally calibrate the enhancement factor that applies to the gas absorption lines.

\section{CONCLUSION}

We have demonstrated a simple technique for intra-cavity absorption spectroscopy giving a path length enhancement of $\sim 500$ in the linear regime of operation for very weak absorption lines or low gas concentrations, transforming a $6 \mathrm{~cm}$ path-length, fiber-coupled, micro-optic cell into an effective path length of 30 meters. The system makes use of the ASE already present within the fiber laser cavity, which also provides a convenient broadband source for the simultaneous interrogation of several gases within the gain-bandwidth of the erbium fiber. Apart from the OSA, all components are inexpensive and the system is very simple to construct and operate. The work reported here is based on erbium fiber lasers, but the principle may be used in other fiber lasers systems where broadband ASE is present along with a flat gain curve and the use of other types of doped fiber lasers or Raman fiber lasers [8] would extend the wavelength region to include several other important gases such as methane and ethane. As well as calibration issues, future work will investigate the use of gain-flattening filters so that the region of maximum enhancement may be tuned across the spectral areas of interest.

\section{REFERENCES}

[1] G. Whitenett, G. Stewart, H. Yu, and B. Culshaw, "Investigation of a tuneable mode-locked fiber laser for application to multipoint gas spectroscopy," J. Lightw. Technol., vol. 22, no. 3, pp. 813-819, Mar. 2004.

[2] V. M. Baev, T. Latz, and P. E. Toschek, "Laser intracavity absorption spectroscopy," Appl Phys B, vol. 69, no. 3, pp. 171-202, Sep. 1, 1999.

[3] G. Stewart, P. Shields, and B. Culshaw, "Development of fibre laser systems for ring-down and intracavity gas spectroscopy in the near-ir," Meas. Sci. Technol., vol. 15, no. 8, pp. 1621-1628, Aug. 1, 2004.

[4] G. Stewart, G. Whitenett, K. Vijayraghavan, and S. Sridaran, "Investigation of the dynamic response of erbium fiber lasers with potential application for sensors," J. Lightw. Technol., vol. 25, no. 7, pp. 1786-1796, Jul. 2007.

[5] A. Stark, L. Correia, M. Teichmann, S. Salewski, C. Larsen, V. M. Baev, and P. E. Toschek, "Intra-cavity absorption spectroscopy with thulium doped fibre laser," Opt. Commun., vol. 215, pp. 113-123, 2003.

[6] Y. Sun, J. L. Zyskind, and A. K. Srivasta, "Average inversion level, modeling, and physics of erbium-doped fiber amplifiers," IEEE J. Sel. Topics Quantum Electron., vol. 3, no. 4, pp. 991-1007, Aug. 1997.

[7] G. Stewart, C. Tandy, D. Moodie, M. A. Morante, and F. Dong, "Design of a fibre optic multi-point sensor for gas detection," Sens. Actuators B, Chem., vol. 51, no. 1-3, pp. 227-232, Aug. 1998.

[8] E. M. Dianov and A. M. Prokhorov, "Medium-power CW Raman fiber lasers," IEEE J. Sel. Topics Quantum Electron., vol. 6, no. 6, pp. 1022-1028, Nov. 2000.

Norhana Arsad received the B.Eng. degree in computer and communication systems and the M.Sc. degree in photonics from Universiti Putra Malaysia (UPM), Malaysia, in 2000 and 2003, respectively, and the Ph.D. degree from the University of Strathclyde, Glasgow, U.K., , in 2010.

She is currently a Lecturer with the Department of Electrical, Electronic and Systems, Universiti Kebangsaan Malaysia (UKM), Bangi, Selangor, Malaysia. Her research interest is in the investigation and design of fiber laser systems for application in spectroscopy, gas sensing, and photonics technology. 
Min Li received the Ph.D. degree in physical electronics from the Fiber Optic Sensing Center, Tsinghua University, Beijing, China, in 1999. Her doctoral work focused on research on fiber optics

She is currently a Professor of physics with the Wuhan University of Technology, Wuhan, China. She was then a Postdoctoral Researcher with the Fiber Optics and Photonics Material Engineering Center, Electrical and Computer Engineering Department, Drexel University, Philadelphia, PA, between 1999 and 2001, and a Senior Engineer with Photonics Laboratories Inc. between 2001 and 2003. She was the primary author of three specialist textbooks and is the author or coauthor of more than 30 technical and scientific papers in the field of fiber optics. Her research interests are widespread over fiber long-period grating sensor and its device fabrication and applications, chalcogenide fiber and devices, multimode and microbending strain sensor, distributed and phase-sensitive OTDR sensors, and trace-element biosensors. The current project she has being working on is trace-element sensing with fiber fluorescent sensor.

George Stewart received the B.Sc. (with First Class Honours) and Ph.D. degrees from the University of Glasgow, Scotland, U.K., in 1974 and 1979, respectively. His doctoral research focused on integrated optics.

He was employed as a Research Fellow with the University of Glasgow until 1985, where he continued research on integrated optics. In 1985 he joined the Photonics Group (now the Centre for Microsystems and Photonics) at the University of Strathclyde, Glasgow, to pursue research on fiber optic components, optical sensors, fiber lasers, and laser spectroscopy. Currently, he is Deputy Head of the Department of Electronic and Electrical Engineering and Professor of photonic engineering with the University of Strathclyde. He is the author or coauthor of more than 150 technical and scientific papers in the field of fiber and integrated optics.

Prof. Stewart is a member of the Optical Society of America and the Institute of Physics.
Walter Johnstone received the B.Sc. (hons.) degree in chemical and material sciences and Ph.D. degree in laser physics from the University of Strathclyde, Glasgow, U.K., in 1977 and 1982, respectively.

Following project engineering and senior technical management posts with Pilkington Optronics Ltd. (now Thales) and Logitech Ltd. (1980-1986), he returned to the University of Strathclyde, where he is Head of Department and a Professor of photonic systems with the Department of Electronic and Electrical Engineering. His research into optical waveguide components, fiber lasers, distributed fiber sensing systems, and optical gas sensing has led to more than 170 technical publications and several patent applications. In addition to his academic career, he has been a director of OptoSci Ltd. since 1994, where he has been instrumental in bringing a number of photonics products to market. 